\title{
NOTA
}

\section{NASTURTIUM OFFICINALE (BRASSICACEAE): NUEVA CITA PARA LA FLORA DEL PARAGUAY}

ROSA DEGEN NAUMANN \& FÁTIMA MERELES HAYDAR²

\begin{abstract}
Summary: Degen Naumann R. \& F. Mereles Haydar. 2015. Nasturtium officinale (Brassicaceae): a new record for the Paraguayan Flora. Bonplandia 24(1): 63-65.

A new generic and specific record for the Paraguayan Flora, Nasturtium officinale W. T. Aiton (Brassicaceae), is documented. This species was found on the streams of Areguá city, Central Department Capital, in Oriental Region of Paraguay. Description, distribution, habitat, phenology, common names and references for uses of this species are presented.
\end{abstract}

Key words: Brassicaceae, Nasturtium officinale, new record, Paraguayan Flora.

Resumen: Degen Naumann, R. \& F. Mereles Haydar. 2015. Nasturtium officinale (Brassicaceae): nueva cita para la Flora del Paraguay. Bonplandia 24(1): 63-65.

Se documenta la presencia de Nasturtium officinale W. T. Aiton (Brassicaceae), nuevo género y especie para la Flora del Paraguay. La misma fue encontrada en los arroyos de la ciudad de Areguá, Capital del Departamento Central en la región Oriental del Paraguay. Se incluyen en este trabajo una descripción breve, comentarios sobre su distribución, el hábitat, la fenología, el nombre vernacular y observaciones sobre su uso.

Palabras clave: Brassicaceae, Flora del Paraguay, Nasturtium officinale, nueva cita.

\section{Introducción}

Nasturtium officinale W. T. Aiton, cuyo nombre vernacular en Paraguay es "berro", es una planta introducida y cultivada, muy difundida en el país y la región por sus usos alimenticios (Martínez Crovetto, 1981). La planta es conocida en algunos sitios del centro de la región Oriental del Paraguay por estar cultivada en grandes piletas, como por ejemplo

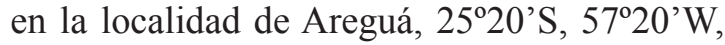
Departamento Central y San Bernardino, $25^{\circ} 20^{\prime} \mathrm{S}, 57^{\circ} 15^{\prime} \mathrm{W}$, Departamento Cordillera, donde se las cosecha para ser utilizada en la alimentación. Mereles (2006) ya la cita como una especie aclimatada; sin embargo no se da ninguna otra referencia de la especie que no sea el uso.

${ }^{1}$ Departamento de Botánica, Dirección de Investigación, Facultad de Ciencias Químicas, Universidad Nacional de Asunción, CC 11001-3291, San Lorenzo, Campus UNA, Paraguay. Investigador PRONII-CONACYT. E-mail: degenrosa@gmail.com; rdegen@qui.una.py

${ }^{2}$ Centro para el Desarrollo de la Investigación Científica (CEDIC). Laboratorios Díaz-Gill y Fundación Moisés Bertoni para la Conservación de la Naturaleza. Manduvirá e/ 15 de Agosto y Juan E. O’Leary. Asunción, Paraguay. 
Nasturtium officinale, es originaria de Europa y Asia central y fue introducida en Paraguay desde mediados del siglo pasado por europeos, dado que la misma es muy apreciada para la alimentación. González Torres (1992) menciona que el "berro" es una planta comestible, con sabor picante y usado en ensaladas, siendo las hojas ricas en vitamina $\mathrm{A}$ $\mathrm{y}$ también en iodo, hierro y vitamina $\mathrm{C}$; como propiedades menciona que es refrescante, diurético, tónico y pectoral y desde hace tiempo es conocida como antiescorbútico. En el Paraguay se la cosecha en los meses más fríos, para lo cual se practica una poda a la parte emergente de la planta (Mereles, 2006).

El objetivo de este trabajo es registrar la presencia de Nasturtium officinale como una especie adventicia para la flora paraguaya.

Nasturtium officinale W. T. Aiton, Hort. Kew. ed. 2, 4: 110. 1812.

Rorippa nasturtium-aquaticum (L.) Hayek, Sched. Fl. Stiriac. 3-4: 22. 1905.

Hierba perenne, enraizada. Raíz fibrosa. Tallos ascendentes, robustos, fistulosos, entre 10-50 cm de altura, algo carnosos, muy ramificados y con raíces adventicias en los nudos sumergidos. Hojas alternas, lámina simple, ovada a suborbicular o más frecuente pinnada, con 1-5 pares de folíolos, elípticos u ovados, bordes lisos a levemente sinuosos de 4-12 cm de long., las terminales más grandes que el par basal. Flores pequeñas agrupadas en racimos terminales, 4 pétalos blancos, 3-5 $\mathrm{mm}$ long. Fruto silicua oblonga, algo arqueada hacia el ápice, 1-2 cm de long., más largo que el pedúnculo.

Fenología: Florece desde setiembre hasta noviembre aproximadamente; fructifica de octubre a diciembre.

Ecología: Hierba acuática, enraizada, semisumergida en aguas lóticas poco profundas, someras, de escaso caudal y sustrato arenoso, con las hojas superiores y las flores completamente emergentes. Forma densas poblaciones. Crece en forma espontánea en los cursos de agua en donde la corriente y la profundidad del agua les son favorables. Frecuentemente se la menciona como una hierba invasora, sin embargo en Paraguay no se comporta de la misma manera y aparentemente su presencia está muy sujeta al comportamiento del agua; de hecho es bastante escasa en el medio natural.

En Paraguay se la cultiva en ambientes de aguas mixtas o semilóticas con escaso caudal, en las denominadas berrerías o parcelas con agua en donde se cultiva al berro. En las mismas se regula el caudal del agua procedente de los arroyos, por medio de un embalse instalado en las cabeceras de las parcelas.

Distribución regional: Es una especie adventicia en Argentina, Brasil, Chile y Uruguay.

Material estudiado: PARAGUAY. Central: Areguá, arroyo Areguá y alrededores, 12-VI2014, Mereles 10031 (CTES, FCQ).

Thellung (1917) mencionan al género Nasturtium W. T. Aiton por primera vez para el Paraguay en base a $N$. bonariense (Poir.) DC. y N. palustre (Leyss.) DC., los cuales son sinónimos de Rorippa bonariensis (Poir.) Macloskie var. bonariensis y $R$. palustris (L.) Besser, respectivamente. También se mencionan para el Paraguay dos especies del género Rorippa Scop. (Al-Shehbaz, 2008), que nada tienen que ver con $N$. officinale, registrada como adventicia.

Sin embargo es interesante mencionar que los géneros Nasturtium y Rorippa son muy afines entre sí (Al-Shehbaz \& Price, 1998), estando ambos presentes en el país.

\section{Bibliografía}

AL-SHEHBAZ, I. A. 2008. Brassicaceae. En ZULOAGA, F., MORRONE, O. \& M. BELGRANO (eds.), Catálogo de las Plantas Vasculares del Cono Sur (Argentina, Sur de Brasil, Chile, Paraguay y Uruguay). Dicotyledoneae: Acanthacease-Fabaceae (Abarema - Schizolobium). Monogr. Syst. Bot. Missouri Bot. Gard. 107: 1691-1692. 
AL-SHEHBAZ, I. A. \& R. A. PRICE. 1998. Delimitation of the genus Nasturtium (Brassicaceae). Novon 8: 124-126.

GONZALEZ TORRES, D. 1992. Catálogo de plantas medicinales (alimenticias y útiles) usadas en el Paraguay. Editorial Imprenta Nacional. 456 pp.

MARTÍNEZ CROVETTO, R. 1981. Las plantas utilizadas en medicina popular en el NO de Corrientes
(República Argentina). Fundación Miguel Lillo, Ministerio de Cultura y Educación. Miscelánea 69: 50.

MERELES, F. 2006. La diversidad, los usos y la conservación de las especies vegetales en los humedales del Paraguay. Rojasiana 7: 171-185.

THELLUNG, A. 1917. Cruciferae. En HASSLER E., Addenda ad Plantas Hasslerianas: 6.

Original recibido el 28 de septiembre de 2014; aceptado el 15 de mayo de 2015. 
